\title{
Psychosocial Experience in Goiter Patients
}

\author{
Spéro H. Raoul Hounkpatin ${ }^{*}$, Fatiou Alabi Bouraima1, Ulrich B. Vodouhe², Omer Adjibode1, \\ Marius Claude Flatin ${ }^{1}$, Prosper Gandaho ${ }^{3}$
}

\author{
${ }^{1}$ Department of Surgery and Surgical Specialties, Faculty of Medicine, University of Parakou, Parakou, Benin \\ ${ }^{2}$ Department of Surgery and Surgical Specialties, Faculty of Sciences and Health, University of Abomey-Calavi, Abomey-Calavi, Benin \\ ${ }^{3}$ Department of Medicine and Medical Specialties, Faculty of Medicine, University of Parakou, Parakou, Benin \\ Email: *speraoul@yahoo.fr
}

How to cite this paper: Hounkpatin, S.H.R., Bouraima, F.A., Vodouhe, U.B., Adjibode, O., Flatin, M.C. and Gandaho, P. (2018) Psychosocial Experience in Goiter Patients. International Journal of Otolaryngology and Head \& Neck Surgery, 7, 367-374.

https://doi.org/10.4236/ijohns.2018.76037

Received: October 28, 2018

Accepted: November 24, 2018

Published: November 27, 2018

Copyright (๑) 2018 by authors and Scientific Research Publishing Inc. This work is licensed under the Creative Commons Attribution International License (CC BY 4.0).

http://creativecommons.org/licenses/by/4.0/

\begin{abstract}
Introduction: Goiter often poses aesthetic disgrace problem. The psychosocial impacts of goiter, in particular in woman may be significant. Objective: studying the psychological and social impacts of the disease in goiter patients within the African context and their consequences on the treatment. Methods: It was a transversal study that took place from May 1to July 31, 2011 in the Collines and Donga departments situated in the center and the north of Benin. The study consisted of a survey conducted through an individual discussion based on questionnaire submitted to goiter patients who accepted to provide their answers. Results: This survey involved 86 patients of which 83 women and 3 men. Their average age was $43.7 \pm 13.41$. Goiter was noticeable in the totality of the patients. $68.6 \%$ of patients declared that they experienced on daily basis the shame caused by the disease. $66 \%$ thought that it was a natural disease; however, $27.9 \%$ believed that it was caused by bewitchment or sorcery. $46.5 \%$ and $37.2 \%$ resorted to modern and indigenous medicine respectively; $16.3 \%$ made no therapeutic move. Despite the psychosocial impacts of the disease, $50 \%$ of the patients rejected surgical intervention possibility. The core motives they raised were lack of financial means (34.8\%), disease recurrence fright (23.3\%), anesthesia fright (16.3\%), and surgical fright (14\%). Conclusion: Despite the psychological impacts of the disease, strong resistance exists probably more on cultural aspect than the motives mentioned by thyroidectomy patients.
\end{abstract}

\section{Keywords}

Goiter, Iodine, Stigmatization, Thyroidectomy, Psychology

\section{Introduction}

Goiter is a volumetric enlargement of thyroid, whatever its cause [1] [2]. Its 
morphology can be diffuse, nodular or multi nodular. It concerns all countries [3] [4]. The majority of patients are asymptomatic [2]. Goiter can be congenital or acquired. Endemic goiter occurs when the disease affects a significant number of people in a given area (5\% of the population or more) [2] [3]. The most important single causal factor is an insufficient intake of iodine [5] [6]. Woman predominance of goiter is well known [7] [8]. This is a noticeable condition because of anterior and superficial localization of thyroid at the level of the neck and one of the problems goiter poses is therefore aesthetic disgrace. This problem is even more important that the disease affects mainly women. In fact, women are more anxious about their appearance than men. The occurrence of goiter may be experienced as a real drama. The psychological impacts of the disease may be significant, especially in Africa where goiter may evolve for several years and reach enormous proportions in absence of treatment, knowing that patients do not visit health facilities for their treatment [9] [10]. This study was conducted in order to assess psychological and social impacts in goiter patients within the African context and the consequences on their treatment.

\section{Methods}

The study took place from May 1 to July 31, 2011 in Benin, in the commune of Bantè, Dassa, Glazoué and Savalou all in the Collines department in the center of Benin and in the commune of Djougou in the Donga department in north-west Benin. These departments are well known in the country as goiter endemic areas. It was a transversal study based on a structured talk with goiter patients met in their houses thanks to communities' relays. Communities' relays are resourceful persons and volunteers who belong to the community and who are recruited by the Ministry of Public Health to facilitate contact between health agents and the community. The study was non-probabilistic and in terms of sampling, the recruitment of goiter patients was exhaustive. This study systematically took into account all anterior-cervical patients who accepted to participate in the survey and in whom the diagnostic of goiter was retained based on cervical exam and classification system of WHO as follow [3]:

Grade 0: thyroid not palpable, or, if palpable, not larger than normal.

Grade 1a: thyroid distinctly palpable and visible with the head in a raised position; the indication is of a thyroid larger than normal, at least, as large as the distal phalanx of the subject.

Grade $1 \mathrm{~b}$ : thyroid easily palpable and visible with the head in a raised position; the grade also includes all patients with a discrete nodule.

Grade 2: thyroid easily visible with the head in a normal position.

Grade 3: goiter visible at a distance.

Grade 4: monstrous goiter.

The discussion was conducted with individual data collection form. The variables studied were those relating to the epidemiological profile of goiter patients, goiter stage according to the WHO classification, patient's knowledge of 
the cause of goiter, feelings experienced at the discovery of goiter, the therapeutic recovery of goiter patients, the impact on their lives, their feeling about their illness, their perception of the behavior of those around them, the disability caused by the disease in their lives and their opinion about the possibility of surgical management of their disease.

The data was processed and analyzed with software Epi info version 3.5.1.

\section{Results}

\subsection{Epidemiological Profile of People Goiter Patients}

Over 86 goiter patients that participated in the survey, three were men and 83 women. The average age of the sample was $43.7 \% \pm 13.41 \% .80 \%$ were married, $9 \%$ as husband and wife, and $11 \%$ divorced.

\subsection{Goiter Classification}

Goiter is clearly visible when the head/neck is in normal position (goiter grade 2 according to WHO classification) in $23.3 \%$ of the sample and it was voluminous, visible from distance (goiter grade 3 according WHO classification) in $76.7 \%$ of the sample.

\subsection{Understanding of Goiter Cause}

Table 1 highlights the breakdown of the survey by the sample understanding of the goiter cause

\subsection{Feelings Experienced at the Discovery of Goiter}

The feelings experienced by the sample at the discovery of goiter are summed up in Figure 1.

\subsection{Therapeutic Remedy for Goiter Patients}

Over 86 of goiter patients of the survey, $40(46.5 \%)$ referred to modern medicine against 32 for indigenous medicine (37.2\%); 14 (16.3\%) stated that they did not make any therapeutic move.

Table 1. Distribution of the investigated according to the knowledge they had of the goiter cause.

\begin{tabular}{ccc}
\hline & Size & Percentage \\
\hline Natural disease & 57 & 66.3 \\
Bewitchment & 22 & 25.6 \\
Incurable disease & 03 & 03.5 \\
Sorcery & 02 & 02.3 \\
Divine Punishment & 02 & 02.3 \\
Total & 86 & 100 \\
\hline
\end{tabular}




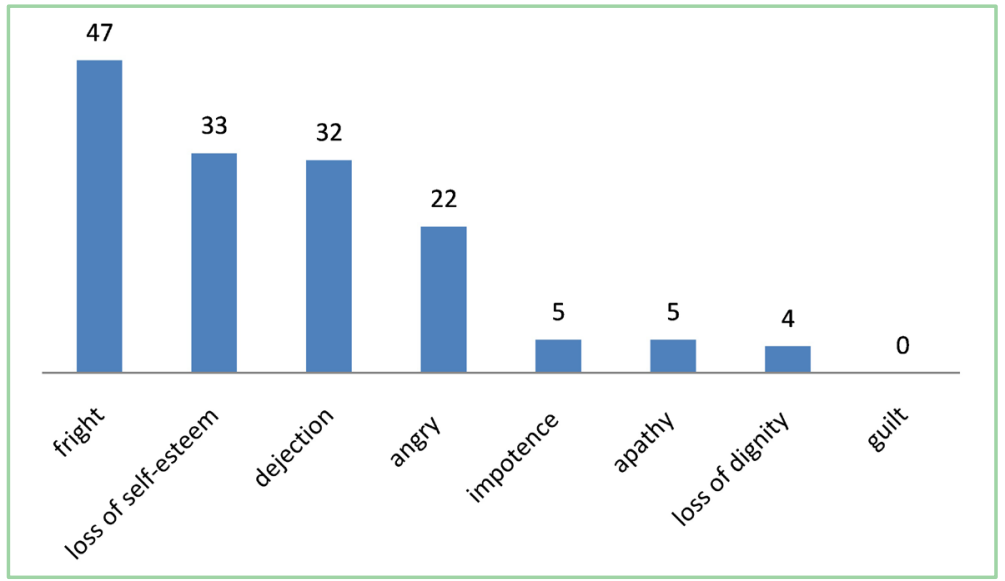

Figure 1. Feeling experienced at the discovery of goiter.

\subsection{Goiter Impacts on Life}

For 16 goiter patients (18.6\%), the disease had no impact on their life, unlike 70 of them $(81.4 \%)$ who stated that goiter affected their life. The feeling they experienced is described in Figure 2.

\subsection{Entourage Behavior}

Table 2 shows the distribution of goiter patients according to their perception on their entourage behavior.

\subsection{Disability in Life}

For 61 goiter patients ( $70.9 \%$ of the sample), the goiter disease was a disability in their life, unlike 25 of goiter patients (29.1\%). Table 3 shows the reasons for which goiter is a disability in goiter patients life of these 61 goiter patients.

\subsection{Quality of Life}

Fifty-four goiter patients (62.8\%) of which the average age is 40.01-year-old declared that they resented the disease whilst 32 goiter patients (37.2\%) of which the average age was 49.94-year-old declared that they fully bear the disease.

The average age of goiter in those who fully resent the disease was 10.72 whilst it was 20.66 in those who fully bear the disease. The means that allow those who fully bear the disease were family support for 53 goiter patients (61.6\%), spouse support for 48 goiter patients (55.8\%) faith for 22 goiter patients $(22.6 \%)$ and friends support for 9 goiter patients (10.6\%). The motives that prevented goiter patients from fully bearing the disease were stigmatization for 55 goiter patients (63.9\%), renunciation or friend's mockery for 30 goiter patients (34.9\%), spouse abandonment for one goiter patients (1.2\%).

\subsection{Surgical Treatment}

Forty-three goiter patients (50.0\%) were against the possibility of surgical treatment. The reasons are in Table 4. 


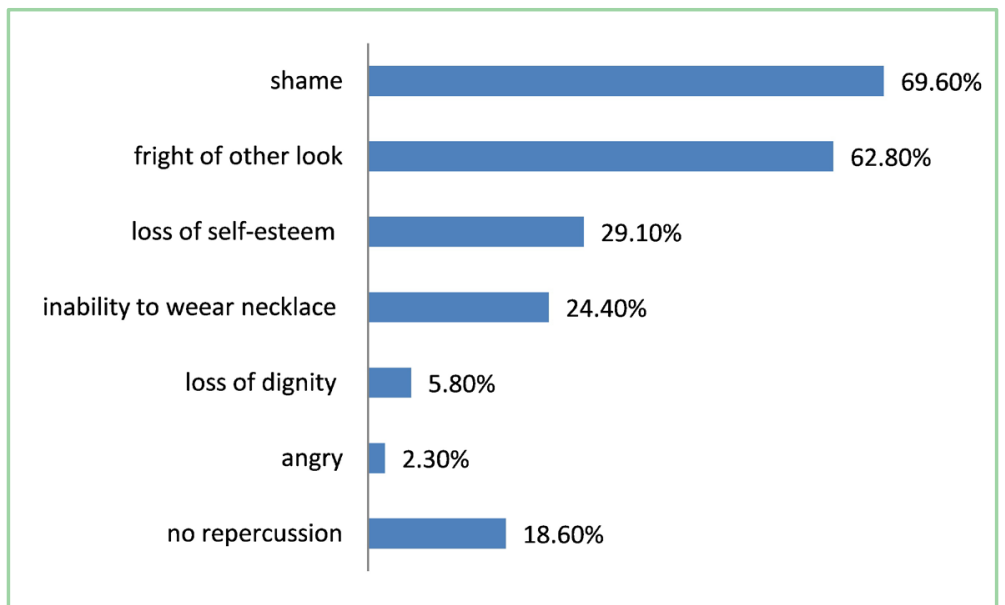

Figure 2. Distribution of goiter patients according to the feelings they had.

Table 2. Distribution of goiter patients according to their perception on their entourage behavior.

\begin{tabular}{ccc}
\hline & Size & Percentage \\
Spouse & 01 & \\
Renunciation & 26 & 01.2 \\
Indifference & 52 & 30.2 \\
Support & 07 & 60.5 \\
Others & & 08.1 \\
Family & 15 & \\
Indifference & 71 & 17.4 \\
Support & & 82.6 \\
Friends & 34 & \\
Indifference & 11 & 39.5 \\
Mockery & 12 & 12.8 \\
Support & 29 & 33.7 \\
Stigmatization & & 14.0 \\
\hline
\end{tabular}

Table 3. Distribution of reasons for which goiter is a disability in goiter patients life.

\begin{tabular}{ccc}
\hline & Size & Percentage \\
\hline Stigmatization & 48 & 78.7 \\
People mockery & 26 & 42.6 \\
Change of clothing habits & 26 & 42.6 \\
Abandonment by spouse & 01 & 1.6 \\
Difficulties in profession performance & 01 & 1.6 \\
\hline
\end{tabular}


Table 4. Distribution of reasons for refusal of surgery by goiter patients.

\begin{tabular}{ccc}
\hline & Size & Percentage \\
\hline Lack of financial means & 15 & 34.8 \\
Recurrence fright & 10 & 23.3 \\
Anesthesia fright & 07 & 163 \\
Surgery fright & 06 & 14.0 \\
Other interference fright & 05 & 11.6 \\
Total & $\mathbf{4 3}$ & 100 \\
\hline
\end{tabular}

\section{Discussion}

The study is not probabilistic and rests essentially on the statements of people with goiter. However, it has the merit to address an aspect of the disease which the caregivers often neglect, particularly surgeons in the treatment process of goiter in Africa. In fact, the health system often focuses on trying to solve problems related to geographical and financial accessibility of the populations to health care, little interest is paid in general to cultural accessibility of the populations to health facilities. Taking into account feeling by goiter patients diagnosed with the disease, it is surprising that some patients made no therapeutic move. Whilst prior to suffering from the disease $66 \%$ of the patients thought that goiter was a "natural disease" and $28 \%$ believed that it was caused by bewitchment or sorcery, the therapeutic care they referred to was the modern medicine and indigenous medicine by $46.5 \%$ and $37.2 \%$ respectively. This reflects the important ratio of the indigenous medicine in our society but this also allows some uncertainty about the reliability of the patients in terms of their statements over their use or reference to indigenous medicine. It is common to notice in common practice that patients lose sight of their use of indigenous medicine while they bear the marks such as scarifications. Different cultural and social interpretations out of diseases generally in Africa could explain this situation. As far as Benin culture is concerned, the role of indigenous healer is dominating in the disease process [11] [12]. He/she is the only person to identify the initial factors of the disease: the cause of the illness (sorcery, divinity, spirit) and historical factor, medium of the disease (transgression of prohibition, relational conflict). The resolution of the problem caused by these two factors is prerequisite to the success of all therapy. The indigenous healer is known as intermediary between spirits, ancestors and people. It is only him/her who is really capable to overcome the disease, to prevent possible complications and to thwart all bad fates. So, despite the disability goiter causes, there is a reluctance of the patients to surgical treatment which, beyond reasons goiter patients put forward, is more cultural. Yet, lack of financial means raised by many of them is a real problem [10] [13]. Nevertheless, it is not insurmountable given the indisputable support of families and spouses reported by several carriers of goiter as well as the various structures of assistance to indigents that exist. Lack of money could not 
therefore be the reason behind systematical refusal of surgery, nor indeed the surgery apprehension itself. First the way in goiter surgery is the neck which is a sensitive area in the patients' culture. Incise the neck as is the chicken equals to life thread cutting risk. Life traditionally is in the neck. General anesthesia is also a source of fright as it is considered as a semi-death state. It is a fragile period for the patients whose way out is unpredictable and who strongly believe in malevolent spiritual forces. These reasons are consistent with problems raised by Amoussou-Guenou et al. in 2005 in Cotonou when he studied problems related to hyperthyroid treatment [14].

\section{Conclusion}

As unsightly phenomenon goiter leads to an alteration of self-image and bad life quality of people with goiter. It is clear that goiter disturbs the setting of relational things and the expected balance in social situations. Despite the considerable psychosocial impact of goiter, strong resistance to surgical treatment exists. This treatment should be multidisciplinary and should not rest on the surgeon alone. It must involve the entire personality of goiter patients with equal focus on their physical, psychological and spiritual state. It is only at this price that we will have the accession of a larger number of goiter patients in surgical treatment within the context of the developing African countries.

\section{Conflicts of Interest}

The authors declare no conflicts of interest regarding the publication of this paper.

\section{References}

[1] Klein, M. and Brunaud, L. (2008) Goitre et nodule thyroidien. La Revue du Praticien, 58, 1249-1259.

[2] Chalari, D., Gerber, F. and Matter, J. (2017) Le goitre en medicine générale. Forum Medical Suisse, 17, 1095-1102.

[3] De Maeyer, E.M., Lowenstein, F.W. and Thilly, C.H. (1979) The Control of Endemic Goitre. World Health Organization. http://apps.who.int/iris/bitstream/10665/40085/1/9241560606_eng.pdf?ua=1

[4] Ogbera, A.O. and Kuku, S.F. (2011) Epidemiology of Thyroid Diseases in Africa. Indian Journal of Endocrinology and Metabolism, 15, 82-88. https://doi.org/10.4103/2230-8210.83331

[5] Brucker-Davis, F., Hieronimus, S. and Fenichel, P. (2016) Thyroïde et environnnement. La Presse Médicale, 45, 78-87. https://doi.org/10.1016/j.lpm.2015.06.015

[6] Delagance, F. (1994) The Disorders Induced by Iodine Deficiency. Thyroid, 4, 107-128. https://doi.org/10.1089/thy.1994.4.107

[7] Sidibé, E.H. (2007) Thyréopathies en Afrique subsaharienne. Santé, 17, 33-39.

[8] Ouédraogo, B.P., Sawadogo, A., Zaghré, N., Goueta, A., Ouattara, M. and Ouoba, K. (2016) Le goitre en ORL: Aspects épidémiologiques, diagnostiques et thérapeutiques. Revue Africaine d ORL et de Chirurgie Cervico-Faciale, 16. http://www.santetropicale.com/manelec/fr/orl/visio_sorlaf.asp?pdf=2016_01_02 
[9] Rumstadt, B., Klein, B., Kirr, H., Kaltenbach, N., Homenu, W. and Schilling, D. (2008) Thyroid Surgery in Burkina Faso, West Africa: Experience from a Surgical Help Program. World Journal of Surgery, 32, 2627-2630. https://doi.org/10.1007/s00268-008-9775-6

[10] Hounkpatin, S.H.R., Lawson-Afouda, S., Avakoudjo, F., Domche Kandem, L. and Adjibabi, W. (2016) Itinéraire thérapeutique des patients ORL dans un Centre Hospitalier régional du Nord-Bénin. La Revue Africaine d ORL et de Chirurgie Cervico-Faciale, 15, 47-52.

www.santetropicale.com/manelec/fr/orl/visio_sorlaf.asp?pdf=2015_02_09

[11] Ouédraogo, D. (2008) La maladie et la manière d'être en Afrique noire. Espace éthique/Ile-de-France.

http://www.espace-ethique.org/ressources/article/la-maladie-et-la-mani\%C3\%A8red\%E2\%80\%99\%C3\%AAtre-en-afrique-noire

[12] Reveyrand, O. (1983) Étiologie et perception de la maladie dans les sociétés modernes et traditionnelles. Premier colloque national d'anthropologie médicale, Paris. http://www.politique-africaine.com/numeros/pdf/016144.pdf

[13] Ouendo, E.M., Makoutodé, M., Paraiso, M.N., Wilmet-Dramaix, M. and Dujardin, B. (2005) Itinéraire thérapeutique des malades indigents au Bénin (Pauvreté et soins de santé). Tropical Medicine \& International Health, 10, 179-186. https://doi.org/10.1111/j.1365-3156.2004.01371.x

[14] Amoussou-Guenou, D., Zannou, D.M., Ade, G., Djrolo, F. and Bigot, A. (2005) Les problèmes liés à la prise en charge de l'hyperthyroïdie à Cotonou. Louvain Medical, 124, 126-131. 